\title{
Triggered star formation in Orion cometary clouds?
}

\section{The case of L1616}

\author{
T. Stanke ${ }^{1, \star}$, M. D. Smith ${ }^{2}$, R. Gredel ${ }^{3}$, and G. Szokoly ${ }^{4}$ \\ 1 Max-Planck-Institut für Radioastronomie (MPIfR), Auf dem Hügel 69, 53121 Bonn, Germany \\ 2 Armagh Observatory, College Hill, Armagh BT61 9DG, Northern Ireland, UK \\ e-mail:mds@star.arm.ac.uk \\ 3 Max-Planck Institut für Astronomie, Königsstuhl 17, 69117 Heidelberg, Germany \\ e-mail: gredel@caha.es \\ 4 Astrophysikalisches Institut Potsdam, An der Sternwarte 16, 14482 Potsdam, Germany \\ e-mail: gpszokoly@aip.de
}

Received 19 April 2002 / Accepted 11 July 2002

\begin{abstract}
We present new $1.2 \mathrm{~mm}$ continuum maps and near- and mid-infrared images of the cometary cloud L1616 located to the west of the Orion $\mathrm{OB}$ associations and apparently shaped by the winds and radiation coming from the massive, hot stars in the $\mathrm{OB}$ association. The new data reveal evidence for ongoing star formation in the cloud in addition to the known cluster of somewhat more evolved stars illuminating the NGC 1788 reflection nebula in the head of the cometary cloud. The $1.2 \mathrm{~mm}$ survey reveals a tight group of dust continuum sources, the brightest of which is seen to drive a powerful near-infrared $\mathrm{H}_{2}$ jet, apparently a very young protostar of Class 0 type. The location of the newly discovered protostar with respect to the older cluster and the direction towards the $\mathrm{OB}$ association suggests an age sequence due to a wave of star formation driven through the cloud and triggered by the impact of the nearby OB association: the older generation of stars is located on the side of the cloud directly facing the $\mathrm{OB}$ association, whereas a new generation of star formation takes place deeper within the cloud.
\end{abstract}

Key words. stars: formation - ISM: clouds - ISM: jets and outflows

\section{Introduction}

A wealth of observations indicates that high mass stars, whether alone or in groups, have a profound impact on the surrounding interstellar material (e.g., Garay \& Lizano 1999; Matzner 2002). The intense, energetic radiation field dissociates and evaporates molecules, atoms, and dust, and together with energetic winds, large bubbles are created surrounding massive stars. The end of their life again has a substantial impact on their surroundings as they explode as supernovae.

The impact of the massive stars on their surroundings might have several consequences. For example, it is thought that star formation in the cloud forming the massive stars itself will be terminated through the action of the radiation field and winds blowing away the parent cloud, thus determining the star

Send offprint requests to: $\mathrm{T}$. Stanke,

e-mail: tstanke@mpifr-bonn.mpg.de

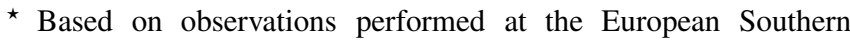
Observatory and the German-Spanish Astronomical Centre, Calar Alto operated by the Max-Planck-Institute for Astronomy, Heidelberg, jointly with the Spanish National Commission for Astronomy. formation efficiency. On the other hand, it is expected that other clouds can be compressed by the radiaton, winds, or supernova shock waves, and thus be triggered to form stars themselves.

Direct evidence for the action of the latter kind of processes seems hard to obtain. Preibisch \& Zinnecker (1999) performed an extensive survey for the low-mass stellar population of the Sco-Cen OB association, and show that the several subgroups have formed at different times, triggered by supernova explosions in the respective older groups. The various subgroups of the Orion $\mathrm{OB}$ association also seem to follow a sequence in age as well as with respect to their location relative to each other, which also hints at a number of star formation events triggered by the others (e.g., Brown et al. 1994).

Going to smaller scales, (Sugitani et al. 1995, 1997, 1999, 2000; Ogura \& Sugitani 1999; Ogura et al. 2002; see also Megeath et al. 1996) have studied a number of "bright rimmed globules" associated with IRAS point sources at optical, IR, and millimetre wavelengths, and there seems to be evidence for an age sequence of young stars dependent on their location with respect to the illuminating massive stars, in a sense that the older stars are located on the side of the cloud facing 


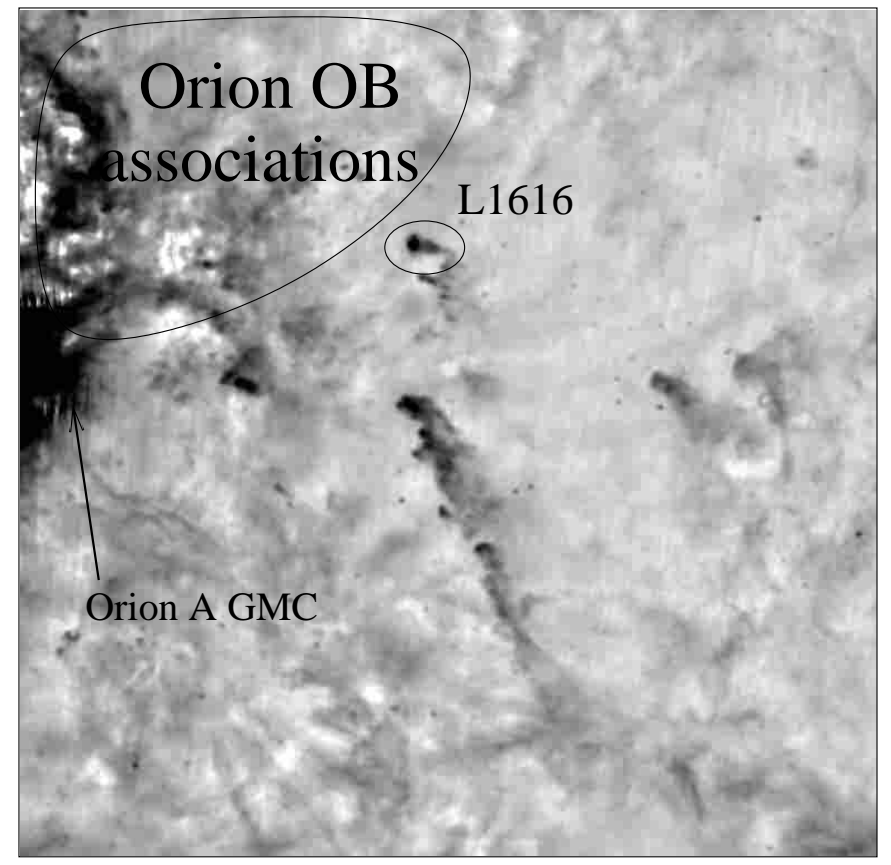

Fig. 1. $100 \mu \mathrm{m}$ IRAS map of a $\sim 15.5 \times 15.5$ area to the southwest of the Orion OB associations. A number of small clouds are visible, which are apparently shaped by various subgroups of the OB association.

the OB stars (or outside the cloud between the cloud and the OB stars), and the younger stars, partly embedded objects, at larger distances from the OB stars. This seems to indicate waves of star formation running through the clouds.

This paper will present a first step in an attempt to extend studies of the kind that Sugitani et al. have presented to the very youngest stars which might form within these clouds and might not even be seen at far-IR wavelengths, e.g., in the IRAS survey data. The goal of this work is to see whether it is possible to locate the actual positions of such waves of star formation propagating through clouds, and to eliminate errors which might occur in the study of the older stars, the "fossil record" of such a wave of star formation because of, for example, uncertain age determinations or by the expected displacement of older stars from the places of their birth.

We have thus initiated a project aimed at studying/searching for Class 0 protostars in a sample of cometary clouds which are apparently shaped by the Orion OB association (see, e.g., Bally et al. 1991; Theil et al. 1995; Ogura \& Sugitani 1998). To do so, we have started to survey these clouds in an unbiased, area covering manner for $1.2 \mathrm{~mm}$ dust continuum sources: Class 0 protostars are generally bright objects at millimetre to submillimetre wavelengths, whereas they are unvisible at optical, near- to mid-infrared wavelengths, and sometimes even at the longest IRAS wavelengths. An unbiased, area covering search for bright millimetre continuum sources is thus the best way to obtain a complete overview of the youngest protostars in the clouds, which is not guaranteed if the protostar searches are restricted to the use of IRAS FIR data or millimetre observations targeted towards IRAS sources (e.g., Sugitani et al. 2000). A second advantage of our survey will be that the target clouds are all in one star forming region, i.e., they can be assumed to be at more or less the same, well known distance (assumed to be about $450 \mathrm{pc}$ in the following). Furthermore, the Orion complex is one of the most nearby OB associations with ongoing star formation in the surrounding clouds, which implies comparatively high spatial resolution as well as sensitivity to faint, low-mass objects.

Here, we present first results of this project, obtained on L1616 (e.g., Ramesh 1995), an otherwise poorly studied cometary cloud east of the Orion A giant molecular cloud. L1616 extends over about 40' ( 5.2 pc at a distance of $450 \mathrm{pc})$ roughly in east-west direction. Its mass as given by Ramesh (1995) is about $157 M_{\odot}$. Its head, pointing towards the east, in the general direction of the Orion OB associations, harbours the NGC 1788 reflection nebula, which is illuminated by a small cluster of low- to intermediate mass stars (see below).

\section{Observations}

\section{1. $1.2 \mathrm{~mm}$ maps}

The MPIfR 37-channel bolometer array MAMBO (Kreysa et al. 1998) was used in Dec. 2000/Feb./Mar. 2001 at the IRAM $30 \mathrm{~m}$ telescope to obtain wide-field, moderate sensitivity maps covering most of L1616 as outlined by its $100 \mu \mathrm{m}$ dust emission. A mosaic of smaller maps was taken, with typical sizes of $16 \times 10$ arcmin (in the so-called fast scan mode, see Teyssier \& Sievers 1999), i.e., applying a faster than standard scan velocity $\left(10^{\prime \prime} / \mathrm{s}\right.$ instead of $\left.4^{\prime \prime} / \mathrm{s}\right)$ and larger than standard elevation steps between individual subscans (22" instead of $\left.4^{\prime \prime}\right)$. The data were reduced in a standard fashion, applying sky-opacity correction, calibration using a counts-to-flux conversion factor derived from maps of bright planets, baseline subtraction, and sky-noise filtering using MOPSI (software package developed by R. Zylka, pers. comm.). As the data were taken in mediocre to rather poor weather conditions, the (chopped) double beam data were not restored to recover the single beam intensity distribution, as this would significantly increase the noise particularly for these rather long (in azimuth) scans. Instead, shiftand-add was applied to use the information contained in the positive and negative beams. Thus, structures greater than the chopper-throw $\left(50^{\prime \prime}\right)$ are suppressed, but compact sources remain unchanged. Finally, the data were averaged and converted from the horizontal system in equatorial maps.

In addition to the wide-field survey data, a smaller map was taken on the only source found in the survey under better weather conditions and using a slower scan speed as well as smaller steps between subscans in order to obtain a good-SN map of this source. In contrast to the wide-field maps, the true single beam intensity distribution was restored from the double beam data, thus compact as well as extended structures are properly recovered.

\subsection{Mid-IR imaging}

We used the TIMMI2 thermal infrared camera on the ESO $3.6 \mathrm{~m}$ telescope on La Silla/Chile on 2001 Jan. 24 and 2001 Dec. 30 to obtain images through an intermediate 


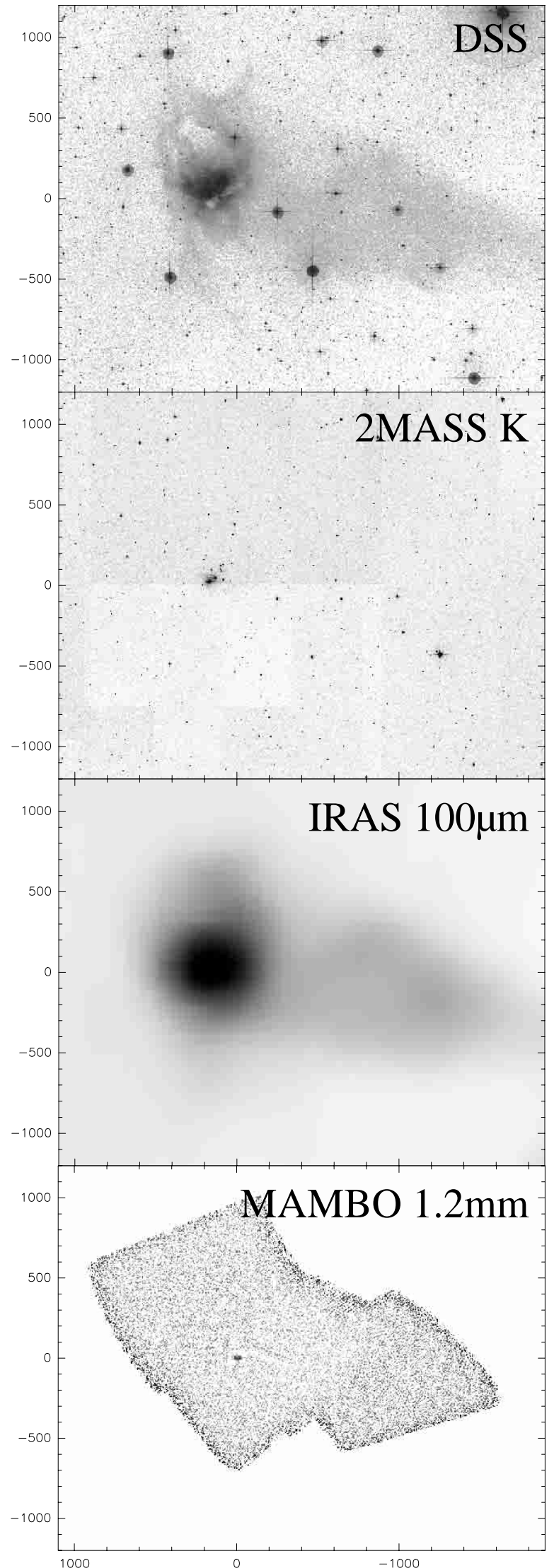

Fig. 2. L1616 at optical wavelengths (a), taken from the Digitized Sky Survey), $K$-band (b), taken from the 2MASS data base), $100 \mu \mathrm{m}$ (c), IRAS), and at $1.2 \mathrm{~mm}$ (d), wide-field fast scan map, this work). Offsets are given in arcseconds from $\alpha=5: 06: 44.4, \delta=-3: 21: 34$ (J2000).

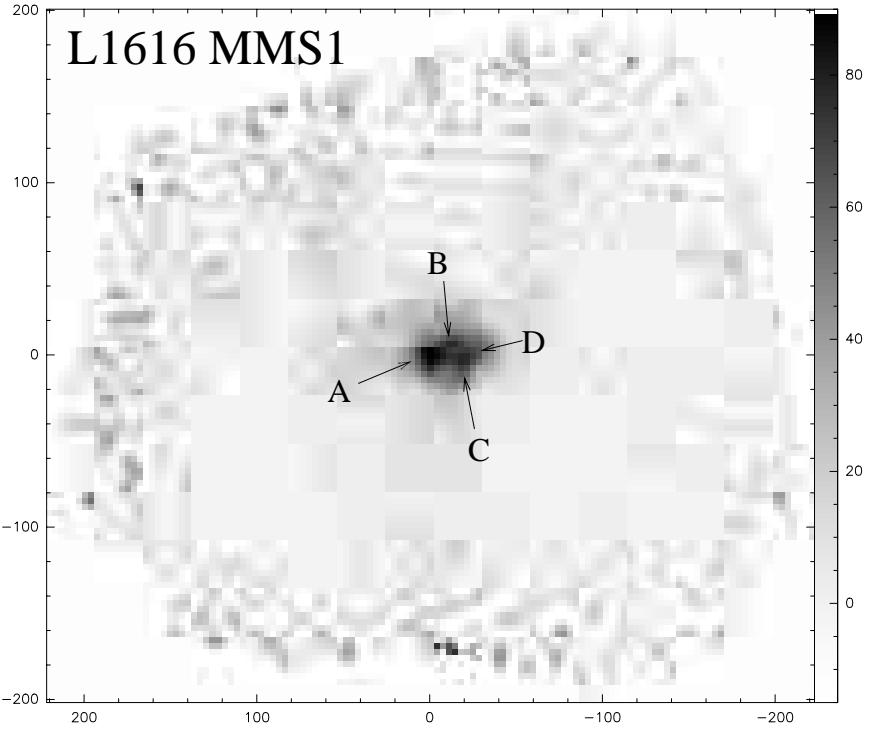

Fig. 3. Higher SN $1.2 \mathrm{~mm}$ map taken towards L1616 MMS1. Offsets are given in arcseconds from $\alpha=5: 06: 44.4, \delta=-3: 21: 34$ (J2000).

passband filter centered at $11.9 \mu \mathrm{m}$ (see www.ls.eso.org/ lasilla/Telescopes/360cat/timmi/for details on the instrument). The observations done in January included one field centered on the newly discovered millimetre source, and a second field centered on the infrared cluster to its north-east. Chopping and nodding of the telescope was done in order to remove the strong thermal background emission of the sky and the telescope, with the chopping in $\mathrm{N}-\mathrm{S}$ direction, and nodding in E-W direction. During the December run, another image was taken centered on the IR cluster, and finally a field was centered to the north-west of the IR cluster, covering the position of the B9V star HD 293815. This time, both chopping and nodding was done in N-S direction.

Data reduction comprised masking of bad pixels and removal of residual background (mostly vertical stripes probably due to instabilities in the readout electronics). The sources are imaged on different parts of the array during the chopping and nodding procedure. In order to make full use of the available information and to improve the signal to noise, the data were shift-and-add combined. The resulting frames were then combined into the mosaic displayed in Fig. 4, weighting them with $1 / \mathrm{rms}^{2}$ (the image displayed in Fig. 4 shows a Gaussian-smoothed version of the final mosaic in order to show the faintest source more clearly).

\subsection{Near-IR imaging}

We used the near-IR camera Omega Prime on the $3.5 \mathrm{~m}$ telescope on Calar Alto/Spain on 2001 Jan. 11 to obtain $2.12 \mu \mathrm{m}$ narrow-band and $K^{\prime}$ wide field images of the region around the newly discovered millimetre source. The data were reduced in a standard fashion, including the subtraction of a sky frame constructed from other science frames and flat-fielding using dome-flats. As these data were taken under poor seeing of $\sim 3^{\prime \prime}$, additional images of the protostellar jet found in the first images were taken in March 2001 using Omega Cass, 


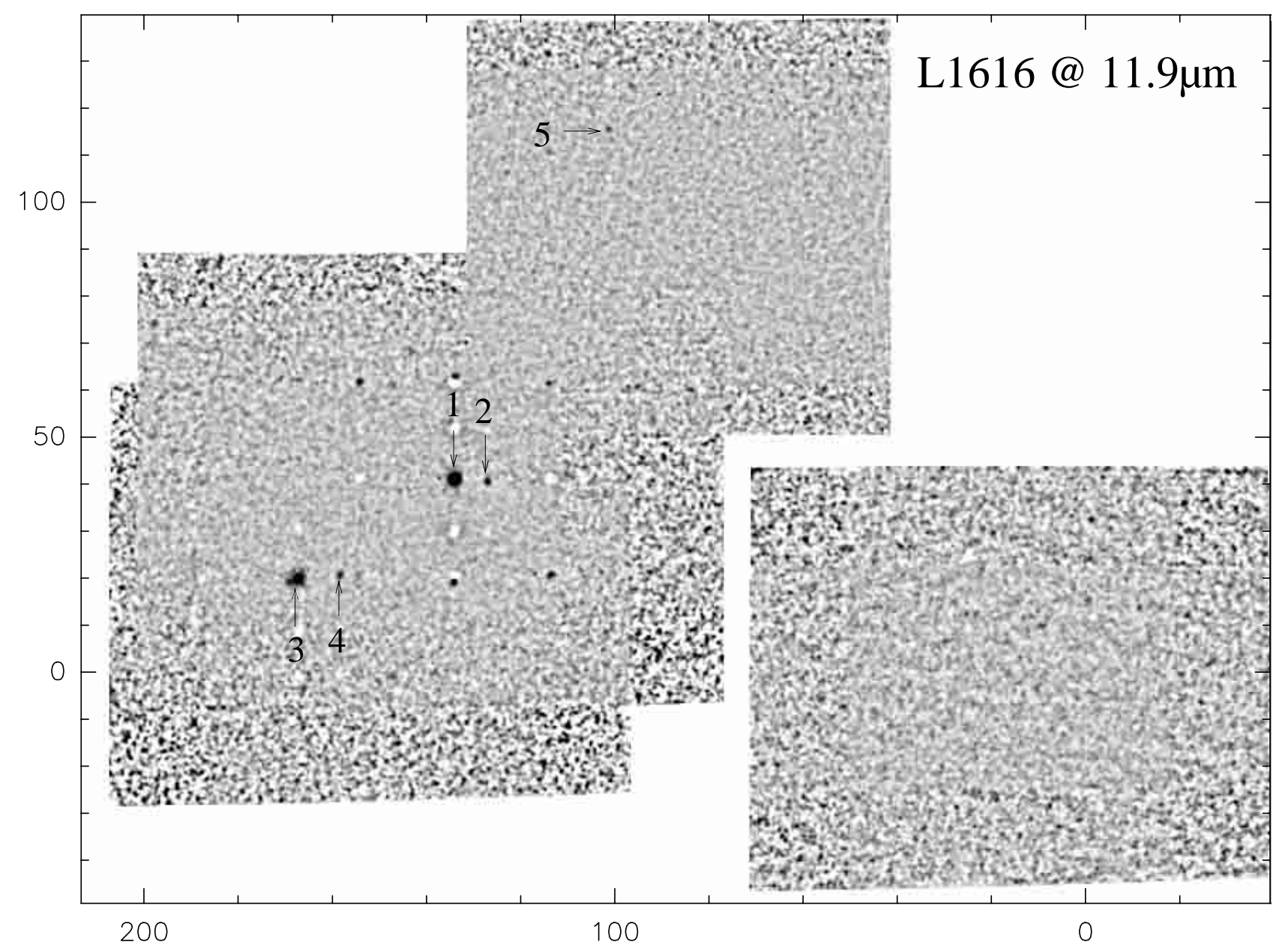

Fig. 4. $11.9 \mu \mathrm{m}$ images taken towards L1616 MMS1. The arrows mark the 5 mid-infrared sources; the remaining positive features as well as the negative features result from the chopping and nodding procedure applied during the observations and subsequent shift-and-add stacking of the data in order to make full use of the available information. Offsets are given in arcseconds from the position of L1616 MMS1 A at $\alpha=$ 5:06:44.4, $\delta=-3: 21: 34$ (J2000). The B9V star HD 293815 (not detected at $11.9 \mu \mathrm{m}$ ) would be located about 1".5 east and $23^{\prime \prime}$ south of MIR5.

also on the Calar Alto $3.5 \mathrm{~m}$ telescope, at a seeing of about $1^{\prime \prime}$ as measured in the $K$-band (seeing at airmass 2-2.5 where observations were taken was about 1.6 arcsec) using a pixel scale of 0.2 (peruse www . caha. es for detailed information on the instruments).

\section{Results}

\section{1. $1.2 \mathrm{~mm}$ mapping}

The wide-field, fast-scan survey of L1616 covered virtually the entire cloud extent as marked by IRAS $100 \mu \mathrm{m}$ emission. One source was found, labeled L1616 MMS1 in the following. The small, higher $S / N$ map targeted on this source clearly resolves L1616 MMS1 into a number of subsources. The brightest peak is located at $\alpha=5: 06: 44.4, \delta=-3: 21: 34$ (J2000; L1616 MMS1 A in the following). At least two, possibly three fainter sources are seen immediately to its west: L1616 MMS1 B located 10" W and 6" N; L1616 MMS1 C located 15" W and 4" S; L1616 MMS1 D located 22" W and $3^{\prime \prime} \mathrm{N}$. The total flux of the group of millimetre sources as measured through an aperture of $77^{\prime \prime}$ is about $1100 \mathrm{mJy}$.
Table 1. Newly discovered $1.2 \mathrm{~mm}$ dust continuum and $11.9 \mu \mathrm{m}$ MIR sources. JHK photometry as well as the positions of the MIR sources are taken from the 2MASS survey.

\begin{tabular}{|c|c|c|c|c|c|c|c|c|c|c|c|}
\hline & \multicolumn{3}{|c|}{ RA (J2000) } & \multicolumn{3}{|c|}{ DEC (J2000) } & \multirow{2}{*}{$\begin{array}{c}F_{1.2 \mathrm{~mm}, \text { peak }} \\
(\mathrm{mJy})\end{array}$} & \multirow{2}{*}{\multicolumn{2}{|c|}{$\begin{array}{c}F_{1.2 \mathrm{~mm}, \text { int }} \\
(\mathrm{mJy})\end{array}$}} & \multirow{2}{*}{\multicolumn{2}{|c|}{$\begin{array}{c}M_{\text {gas }, 15 \mathrm{~K}} \\
\left(M_{\odot}\right)\end{array}$}} \\
\hline & $\mathrm{h}$ & $\mathrm{m}$ & s & $\circ$ & ' & $"$ & & & & & \\
\hline L1616 MMS1 A & 5 & 06 & 44.4 & -3 & 21 & 34 & 95 & & 7 & 0.95 & \\
\hline L1616 MMS1 B & 5 & 06 & 43.7 & -3 & 21 & 28 & 83 & & 5 & 0.2 & \\
\hline L1616 MMS1 C & 5 & 06 & 43.4 & -3 & 21 & 38 & 85 & & 1 & 0.27 & \\
\hline \multirow[t]{3}{*}{ L1616 MMS1 D } & 5 & 06 & 42.9 & -3 & 21 & 31 & 70 & & 8 & 0.30 & \\
\hline & \multicolumn{3}{|c|}{ RA (J2000) } & \multicolumn{3}{|c|}{ DEC (J2000) } & $F_{11.9 \mu \mathrm{m} \quad \mathrm{n}}$ & $\mathrm{m}_{11.9}$ & $J$ & $H$ & $\bar{K}$ \\
\hline & $\mathrm{h}$ & m & & $\circ$ & & $"$ & $(\mathrm{mJy})$ & & & & \\
\hline L1616 MIR1 & 5 & 06 & 53.3 & -3 & 20 & 53 & 800 & 4.0 & 10.4 & 8.9 & 8.1 \\
\hline L1616 MIR2 & 5 & 06 & 52.9 & -3 & 20 & 53 & 180 & 5.6 & 10.0 & 8.8 & 8.4 \\
\hline L1616 MIR3 & 5 & 06 & 55.5 & -3 & 21 & 13 & 300 & 5.0 & 9.9 & 9.1 & 8.5 \\
\hline L1616 MIR4 & 5 & 06 & 54.9 & -3 & 21 & 13 & 130 & 5.9 & 13.0 & 11.0 & 9.5 \\
\hline L1616 MIR5 & 5 & 06 & 51.0 & -3 & 19 & 38 & 85 & 6.4 & 10.8 & 10.0 & 9.6 \\
\hline
\end{tabular}

We also attemted to decompose this total flux into the fluxes of the individual subsources. This was done by fitting four twodimensional Gaussians with FWHMs of the order 10 to $15^{\prime \prime}$ representing the individual subsources, plus one more extended 
Gaussian to model a more extended emission component, apparently a compact core or halo. The resulting fluxes for the four subsources are given in Table 1 in the column labeled as $F_{1.2 \mathrm{~mm} \text {,int }}$. It has to be noted that these fluxes should be regarded as very crude estimates at most, as the sources are separated by only a bit more than one beamwidth, and as the SN in the map is not terribly good.

As thermal dust emission in the (sub)millimetre wavelength range can usually be assumed to be optically thin, the measured $1.2 \mathrm{~mm}$ fluxes can be converted into circumstellar dust masses (e.g., Hildebrand 1982). Assuming a dust temperature of $15 \mathrm{~K}$ and a standard ratio of gas to dust mass of 100, the standard mm-flux to gas mass conversion formula reads

$M_{\mathrm{gas}, 15 \mathrm{~K}}=4.38 \times 10^{-3} M_{\odot} \cdot\left(\frac{F_{v}}{\mathrm{mJy}}\right)\left(\frac{D}{450 \mathrm{pc}}\right)^{2}\left(\frac{0.01 \frac{\mathrm{cm}^{2}}{\mathrm{~g}}}{\kappa_{1.3 \mathrm{~mm}}}\right)$.

For a dust temperature of $10 \mathrm{~K}$ this value has to be multiplied by 1.89 , for a dust temperature of $20 \mathrm{~K}$ by a factor of 0.67 .

The dust masses derived for the individual subsources are given in Table 1 (where a dust temperature of $15 \mathrm{~K}$, a distance of $450 \mathrm{pc}$, and a dust opacity coefficient of $0.01 \mathrm{~cm}^{2} / \mathrm{g}$ was adopted as usually taken for circumstellar envelopes in very young sources; e.g., Motte et al. 1998). The $1.2 \mathrm{~mm}$ flux for the whole group of sources of $1100 \mathrm{mJy}$ translates into a gas mass of about $5 M_{\odot}$. The most massive of the subsources (L1616 MMS1 A) appears to have a circumstellar gas mass of around $1 M_{\odot}$, whereas the other three components seem to be subsolar mass objects.

\subsection{MIR imaging}

A total of five sources were detected at flux levels between $\sim 85$ and 800 mJy (L1616 MIR1-L1616 MIR5; see Fig. 4, Table 1). MIR1 to MIR4 are located in the field centered on the cluster illuminating the reflection nebula NGC 1788 and can be identified with 4 bright NIR sources. The fifth source appears to coincide with a star located due north of the B9V star HD 293815, which itself is not detected to a flux limit of about $80 \mathrm{mJy}$. We do not see any source at the position of the $1.2 \mathrm{~mm}$ source L1616 MMS1 to a flux limit of about $120 \mathrm{mJy}$.

\subsection{NIR imaging}

\subsubsection{The $\mathrm{H}_{2}$ jet from $\mathrm{L} 1616 \mathrm{MMS1}$}

A comparison of our $K^{\prime}$ wide-band and $2.12 \mu \mathrm{m}$ narrow-band images of the area around L1616 MMS1 lead to the identification of three groups of molecular hydrogen emission line features. One feature is closely related to the reflection nebulosity surrounding the brightest members of the cluster illuminating NGC 1788. The most likely explanation for this feature is fluorescent emission from UV excited $\mathrm{H}_{2}$ in the vicinity of the most massive and hottest members of the cluster.

The other two groups of features very clearly form two bow-shock structures to the north-west and to the southeast of the newly discovered L1616 MMS1. Apparently, they are shocks in terminating working surfaces of a protostellar outflow driven by one of the subsources of L1616 MMS1 (we consider the most massive source L1616 MMS1 A as the most likely driving source; no further $\mathrm{H}_{2}$ emission was detected beyond the two bow-shocks on the wide-field images taken with OMEGA prime). The projected length of the outflow (comprising both lobes) is $190^{\prime \prime}$, corresponding to $0.41 \mathrm{pc}$ at a distance of $450 \mathrm{pc}$. The position angle of the outflow is $122^{\circ}$ east of north. The tips of the bow shocks appear to be located virtually symmetric about the location of the driving source, $82^{\prime \prime}$ east $/ 51^{\prime \prime}$ south and $80^{\prime \prime}$ west $/ 49^{\prime \prime}$ north of the driving source, respectively.

\subsubsection{Emission line stars and the embedded IR cluster}

The more evolved population of stars is represented by a handful of emission line stars seen in the head of the globule and a partly embedded cluster of intermediate luminosity sources illuminating NGC 1788. Five emission line objects have been found in L1616 by Nakano et al. (1995; Kiso A-0974 14-18). Three of them (Kiso A-0974 16, 17, and 18) are located to the east and south-east of the center of the reflection nebula NGC 1788; they are of stellar nature and show relatively weak $\mathrm{H} \alpha$ emission. A fourth stellar emission line source is seen just north to the center of the reflection nebulosity (Kiso A-0974 14; $\mathrm{LkH} \alpha 333$; HBC 82) and is of intermediate strength $\mathrm{H} \alpha$ emission. The fifth object (Kiso A-0974 15) has been characterized as non-stellar by Nakano et al.; its position is within the uncertainties coincident with MIR3. MIR3 might thus also be an emission line star, or the $\mathrm{H} \alpha$ emission from Kiso A-0974 15 might indicate a small HII region around MIR3.

Our new near-infrared images reveal a group of bright sources at the heart of NGC 1788, part of which are obscured at optical wavelengths. Four of them can be identified with the four mid-infrared sources MIR1 to 4 noted above. They are surrounded by a small $K$-band reflection nebulosity, which is also associated with (presumably fluorescent) $\mathrm{H}_{2}$ emission. In contrast, the optically visible B9V star HD 293815 is not seen to be directly associated with IR reflection nebulosity nor MIR emission. This suggests that MIR1 to 4 are the main luminosity sources illuminating NGC 1788. Furthermore, the presence of fluorescent $\mathrm{H}_{2}$ emission suggests that at least one of them is also of fairly early spectral type, presumably of mid-B type.

The near- to mid-infrared colours of the 5 MIR sources (see Fig. 6) strongly suggest the presence of warm (a few hundred $K$ and more) circumstellar material in the immediate vicinity of the stars, most likely in the form of circumstellar disks. Although the near-infrared colours might in principle be explained through (partly heavily) reddened stellar photospheres, the $K-N$ colours are not possible to explain this way. However, with the present data it is not possible to put reasonable constraints on the parameters of the disks or to further constrain the masses or spectral types of their central sources; more sensitive data at mm wavelengths (disk masses!) and particularly mid- to far-infrared wavelengths are necessary to constrain the disk properties and the luminosity of the individual sources. 

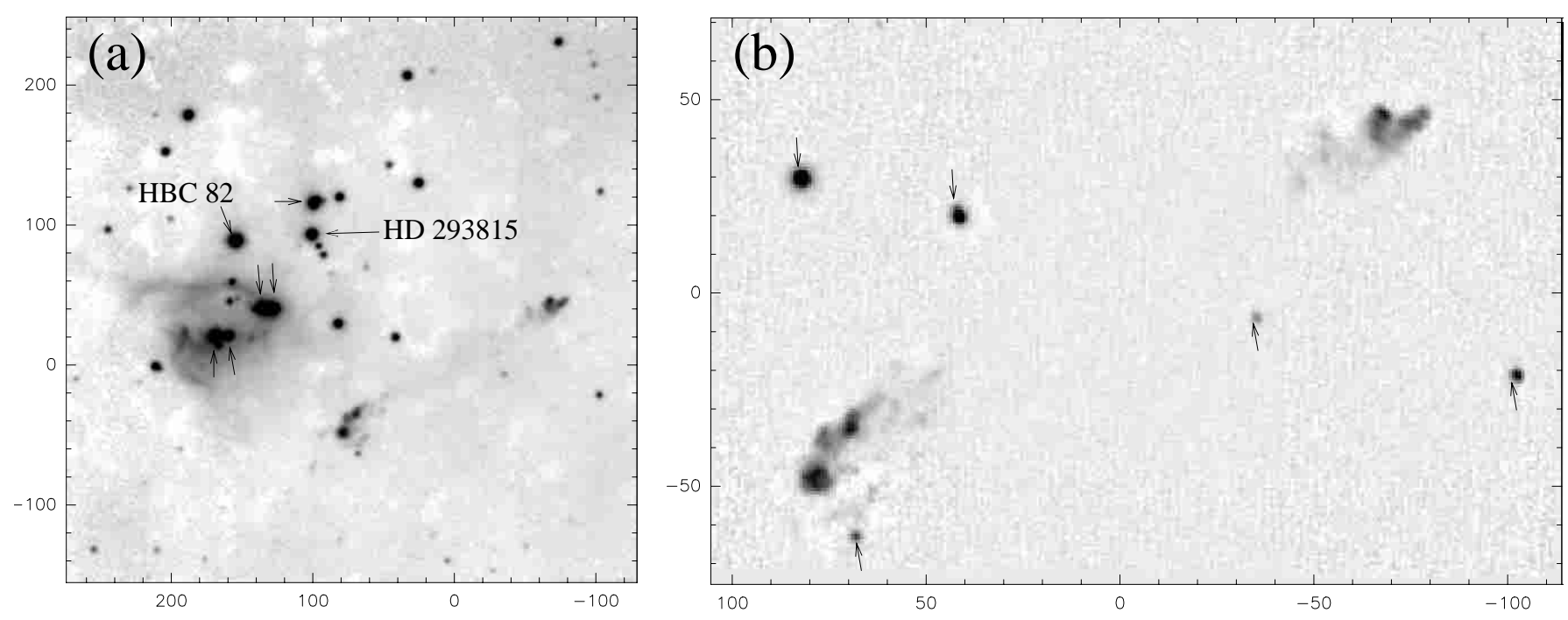

Fig. 5. 2.12 $\mu \mathrm{m}$ narrow-band images (not continuum subtracted) taken towards L1616 MMS1. The small arrows in panel a) mark the midinfrared sources identified in Fig. 4. The arrows in panel b) mark stellar (continuum) sources. Offsets are given in arcseconds from $\alpha=$ 5:06:44.4, $\delta=-3: 21: 34(\mathrm{~J} 2000)$.

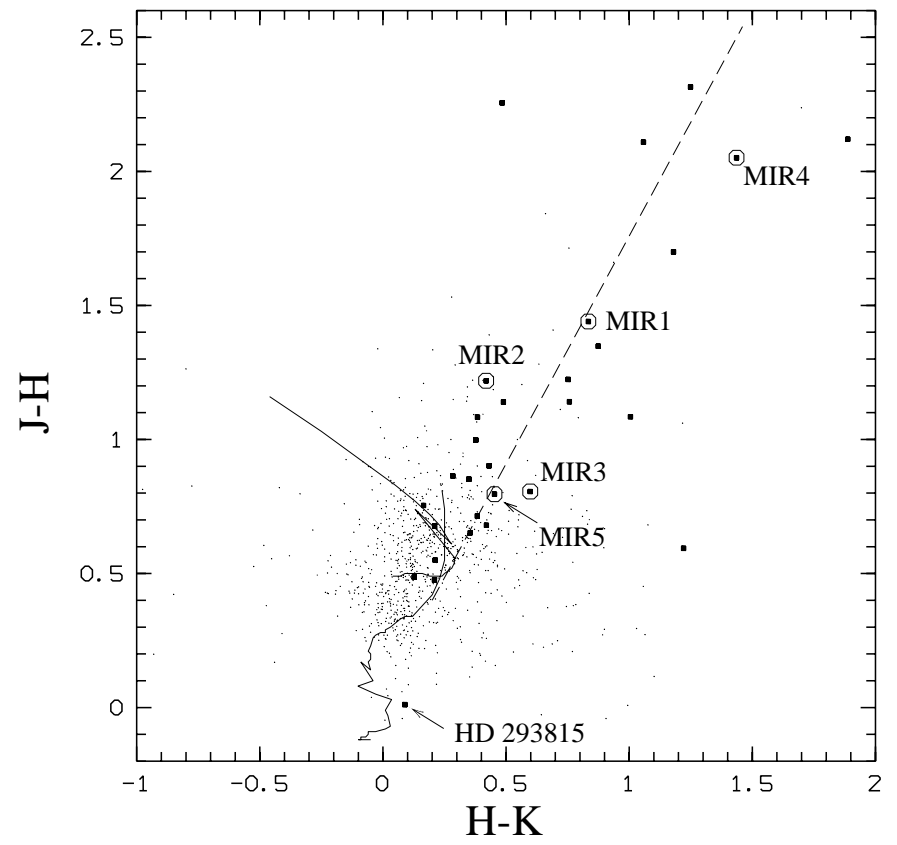

Fig. 6. Colour-colour diagram showing the 2MASS sources in the field around L1616. Small dots represent all sources found in a 34' diameter circle around the cloud head, big dots represent sources in a $7^{\prime}$ circle around the approximate center of the IR cluster, and encircled bid dots mark the 5 newly discovered MIR sources in the IR cluster. The location of unreddened main sequence and giant stars are marked by solid lines, the reddening vector is indicated by the dashed line.

\section{Discussion}

The data presented in this paper show clear evidence for recent as well as ongoing star formation in L1616. The generation of ongoing star formation is represented by L1616 MMS1 A. The data suggest that MMS1 A is a very young protostar, presumably a Class 0 source: these objects are deeply embedded and obscured at optical, near-infrared, mid-infrared, and sometimes even far-infrared wavelengths, but bright at (sub)millimetre wavelengths. These are exactly the characteristics of MMS1 A: it is seen only at $1.2 \mathrm{~mm}$, not detected in our mid-infrared images (which go by a factor of about 2-3 deeper than the IRAS $12 \mu \mathrm{m}$ measurements), and also not seen by IRAS at longer wavelengths. Although this is in part certainly due to the close vicinity of the FIR-bright cluster at the head of the cloud, this underlines the importance of millimetre dust continuum surveys to reveal the youngest protostars, which might be missed even by the IRAS survey. The close vicinity of the FIR bright cluster prevents any reasonable determination of the ratio $L_{\mathrm{submm}} / L_{\mathrm{bol}}$, which is one of the defining criteria for Class 0 sources (e.g., André et al. 1993). But the detection at millimetre wavelengths and the nondetection at near- to mid-infrared wavelengths alone argues for a large value of $L_{\text {submm }} / L_{\mathrm{bol}}$ indicating the probable Class 0 nature of MMS1 A.

A second argument in favour of the great youth of L1616 MMS1 A is the presence of the infrared $\mathrm{H}_{2}$ jet $^{1}$. It appears to be quite short, but fairly bright in $\mathrm{H}_{2}$ emission, which is a particular characteristic of very young flows driven by protostars in the main accretion phase, early during their lives: these outflows have a very active driving source, providing great amounts of energy, thus being able to generate bright shocks, but have not yet had the time to expand to a great length, such as the giant Herbig-Haro outflows seen in many star forming regions (Stanke 2000). The bows are located $\sim 0.20-0.21 \mathrm{pc}$ in projection from the central source, and can be reached in just $2000 \mathrm{yr}$ at $100 \mathrm{~km} \mathrm{~s}^{-1}$.

An earlier generation of star formation yielded the small cluster embedded in the head of the globule, illuminating the reflection nebula. Although the present data are not sufficient to estimate an age for the cluster, it seems that it is fairly young, as there are a number of sources showing substantial

\footnotetext{
${ }^{1}$ Unpublished $\mathrm{CO}(1-0)$ test spectra taken recently by us at the IRAM $30 \mathrm{~m}$ telescope also show the presence of a $\mathrm{CO}$ molecular outflow.
} 


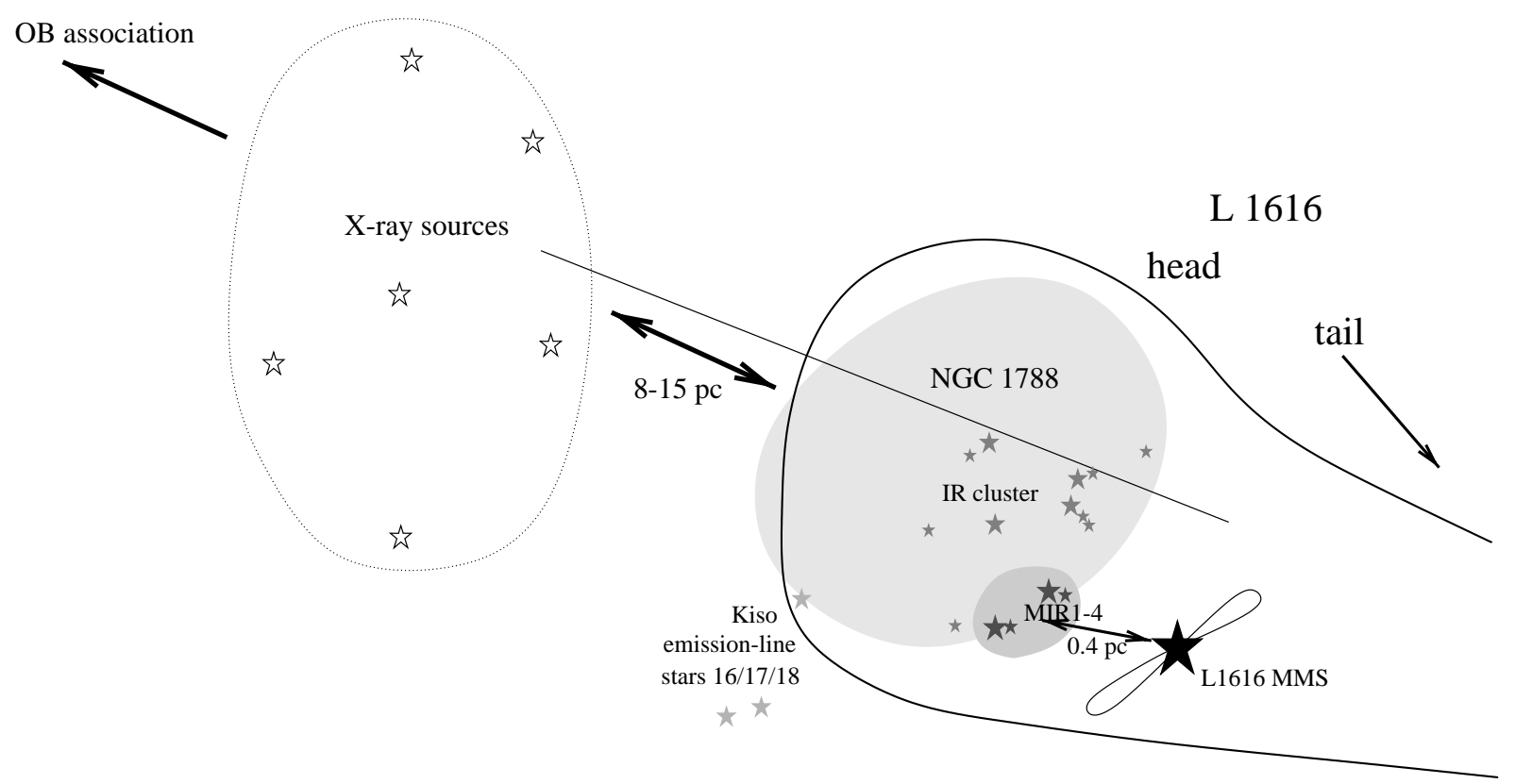

Fig. 7. Schematic drawing of the area under discussion (not to scale).

infrared excess emission at near-infrared as well as at midinfrared wavelengths, indicating the presence of circumstellar disks in at least some of the systems (see Fig. 6). To get at least a very rough idea about the age of the cluster it is interesting to note that the optically visible B9V star HD 293815 has colours consistent with an at most very slightly reddened main sequence star. The pre-main sequence evolution of a star of this spectral type would be finished after a bit less than $10^{6}$ years, thus as a lower limit on the age of the cluster a value of around $10^{6}$ years is reasonable. The detection of mid-infrared excess emission from the stars at the center of the reflection nebulosity in turn very likely implies that these stars are not very much older than $10^{6}$ years, if at all, thus the age of the IR cluster might be around $10^{6}$ years.

To extend further the speculations about the ages of the stellar populations, it is interesting to have another look at the three emission-line stars Kiso A-0974 16/17/18. As noted above, they are located to the east and south-east of the IR cluster; in fact, 17 and 18 are projected just on the leading edge of the cloud. These three stars possess only fairly low-level $\mathrm{H} \alpha$ emission: it might be possible that these stars represent an even older generation of stars, which are already approaching the weak-line T Tauri stage. Finally, it should be noted that a concentration of X-ray detected weak-line T Tauri stars has been claimed to exist $\sim 1-2^{\circ}$ ahead of the cloud (Sterzik et al. 1995; Alcalá et al. 1996). These stars might be part of the oldest population of stars which formed from L1616.

The large displacement of these stars from the actual location of L1616 might have two possible reasons. The first, simplest would be that they don't have any relation to L1616, but formed from a different, now dispersed cloud far ahead of L1616. As a second, L1616 as we see it now might be the last remnant of an initially much larger cloud complex filling the space between the $\mathrm{OB}$ association and the actual location of L1616. From this much larger cloud, the weak line T Tauri stars might have formed (triggered by the impact of the just formed OB stars), and subsequently, the cloud has been destroyed by the impact of the new OB association, with L1616 forming the very last remnant of this cloud. Further data are required to establish a more complete overview of the stellar population in, ahead, and around the cloud, as well as the kinematics of the stars and of the cloud.

This may also help to decide whether star formation has been going on in a continuous fashion in L1616 or as a sequence of burstlike events with quiet periods in between, as might be the case if several supernovae in the Orion OB association had driven shock waves through the cloud. E.g., could it be the case that the embedded Class 0 source was triggered by a recent supernova, and the older IR cluster by an earlier event? Assuming that both objects were triggered by the same event, the separation between the objects and their age difference of 1 to a few $10^{5}$ years would imply a velocity of progression through the cloud of the order of a few tenths of a $\mathrm{km} \mathrm{s}^{-1}$. In contrast, Preibisch \& Zinnecker (1999) argued that the supernova shocks responsible for triggering star formation in the various subgroups in Sco-Cen were progressing much faster, with about $10 \mathrm{~km} \mathrm{~s}^{-1}$. However, the supernova shocks in Sco-Cen presumably were running through a thin intercloud medium and then through a presumably not very dense molecular cloud (maybe $10^{2}$ to $10^{3} \mathrm{~cm}^{-3}$ ), which then formed the stars. In contrast, L1616 is apparently a cometary cloud which has been compressed by the radiation from the OB associations (e.g., Bertoldi 1989; Kessel-Deynet \& Burkert 2002), most likely already before a supposed supernova shock responsible for triggering the IR cluster and Class 0 protostar impacted on the cloud. Thus it may well be the case that a shock wave initially running at (say) $10 \mathrm{~km} \mathrm{~s}^{-1}$ slows down considerably to a value consistent with the few tenths of a $\mathrm{km} \mathrm{s}^{-1}$ in such a dense cloud. 
To summarize, there seems to be evidence for an age sequence of young stars associated with L1616, with the age of stars decreasing with increasing distance from the Orion OB association. The oldest population might be represented by a number of X-ray detected weak-line $\mathrm{T}$ Tauri stars to the east of L1616, followed by the emission line stars found at the eastern edge of L1616. The next generation is represented by the partly embedded cluster illuminating NGC 1788 in the head of the globule, and the youngest objects, the deeply embedded protostellar millimetre sources, are found to the west of NGC 1788.

Compared with the star formation event creating the infrared cluster at the head of the globule (producing at least one, probably several stars of intermediate mass) the youngest group of protostars consists of only low mass objects and apparently does not represent the most productive star formation episode in L1616. More surprising is that, rather than seeing a single protostellar object, a small group of low mass protostars or prestellar dust clumps is detected. Usually the formation of groups or clusters is found to accompany intermediate to high mass star formation rather than low mass star formation. Notably, a number of jet-driving protostars in the solar mass range in Orion appear as single sources when observed at $\mathrm{mm}$ wavelengths with similar angular resolution, possibly surrounded by some diffuse, extended emission, but not as tight groups of separate sources. Indeed it has very recently been argued that collapse induced by compression waves might favour fragmentation of collapsing cores (Hennebelle et al. 2002), thus the newly discovered multiple millimetre source might be additional evidence for triggered star formation in L1616.

Together this suggests that in L1616 we see another example of the action of sequential star formation induced by the interaction of high-mass stars with a cloud, as has been reported by others. However, regarding the protostellar source L1616 MMS1, a second possibility should be kept in mind: the star formation associated with L1616 MMS1 might actually not have been triggered by the impact of the Orion OB associations itself, but rather be a consequence of triggered star formation initiated by the formation of the older cluster of stars illuminating NGC 1788. These stars apparently also do have a substantial impact on the surrounding material as can be seen from the large extent of this reflection nebula, whose shape resembles a large, roughly north-south oriented bipolar cavity which might have been created by molecular outflows during the formation of the cluster.

In order to resolve the issues about triggered star formation, clearly more observations are required. (Sub)Millimetre bolometer arrays will play a crucial role in this game, as it is now finally possible to scan substantial areas of clouds with sufficient sensitivity in a reasonably short amount of time to perform unbiased searches for the youngest protostars in molecular clouds. Thus the locations of present day star formation will be revealed in an unprecedented manner, as demonstrated in this paper.

Acknowledgements. We thank the staff at the IRAM $30 \mathrm{~m}$, at ESO, and on Calar Alto for their support. Part of this work was supported by the Deutsche Forschungsgemeinschaft, DFG project number $\mathrm{Zi} 17 / 2-1$. MDS was was partly supported by INTAS grant 00-00287.

\section{References}

Alcalá, J. M., Terranegra, L., Wichmann, R., et al. 1996, A\&AS, 119, 7

André, P., Ward-Thompson, D., \& Barsony, M. 1993, ApJ, 406, 122

Bally, J., Langer, W. D., Wilson, R. W., Stark, A. A. \& Pound, M. W. 1991, in Fragmentation of Molecular Clouds and Star Formation, ed. I. Falgarone, F. Boulanger, \& G. Duvert (Kluwer), IAU Symp., 147, 11

Bertoldi, F. 1989, ApJ, 346, 735

Brown, A. G. A., de Geus, E. J., \& de Zeeuw, P. T. 1994, A\&A, 289, 101

Ducati, J. R., Bevilacqua, C. M., Rembold, S. B., \& Ribeiro, D. 2001, ApJ, 558, 309

Garay, G., \& Lizano, S. 1999, PASP, 111, 1049

Hennebelle, P., Whitworth, A. P., Gladwin, P. P., \& André, P. 2002, MNRAS, in press [astro-ph/0206044]

Hildebrand, R. H. 1983, Royal Astron. Soc. Quart. J., 24, 267

Kessel-Deynet, O., \& Burkert, A. 2002, MNRAS, submitted [astro-ph/0203460]

Kreysa, E., Gemuend, H.-P., Gromke, J., et al. 1998, Proc. SPIE, 3357, 319

Matzner, C. D. 2002, ApJ, 566, 302

Megeath, S. T., Cox, P., Bronfman, L., \& Roelfsema, P. R. 1996, A\&A, 305, 296

Motte, F., André, P., \& Neri, R. 1998, A\&A, 336, 150

Nakano, M., Wiramihardja, S. D., \& Kogure, T. 1995, PASJ, 47, 889

Ogura, K., \& Sugitani, K. 1998, PASA, 15, 91

Ogura, K., \& Sugitani, K. 1999, in Star Formation 1999, Proc. of Star Formation 1999, ed. T. Nakamoto, 381

Ogura, K., Sugitani, K., \& Pickles, A. 2002, AJ, 123, 2597

Preibisch, T., \& Zinnecker, H. 1999, AJ, 117, 2381

Ramesh, B. 1995, MNRAS, 276, 923

Stanke, T. 2000, Ph.D. Thesis, Universität Potsdam

Sterzik, M. F., Alcalá, J. M., Neuhäuser, R., \& Schmitt, J. H. M. M. 1995, A\&A, 297, 418

Sugitani, K., Morita, K.-I., Nakano, M., \& Tamura, M. 1997, ApJ, 486, L141

Sugitani, K., Tamura, M., \& Ogura, K. 1995, ApJ, 455, L39

Sugitani, K., Tamura, M., \& Ogura, K. 1999, in Star Formation 1999, Proc. of Star Formation 1999, ed. T. Nakamoto, 358

Sugitani, K., Matsuo, H., Nakano, M., Tamura, M., \& Ogura, K. 2000, AJ, 119, 323

Teyssier, D., \& Sievers, A. 1999, IRAM Newslett., 41, 12

Theil, D. S., Yu, K. C., \& Bally, J. 1995, Poster presented at: CO: Twenty-five Years of Millimeter-wave Spectroscopy, Poster Session, IAU, 170 